\title{
B.R.S. Algebras. Analysis of the Consistency Equations in Gauge Theory
}

\author{
M. Dubois-Violette ${ }^{1}$, M. Talon ${ }^{2}$, and C. M. Viallet ${ }^{2}$ \\ 1 Laboratoire de Physique Théorique et Hautes Energies, L.A. 063, Université Paris Sud, \\ Bâtiment 211, F-91405 Orsay, France \\ 2 Laboratoire de Physique Théorique et Hautes Energies, L.A. 280, Université Paris VI, 4 Place \\ Jussieu, F-75230 Paris Cedex 05, France
}

\begin{abstract}
We compute all possible anomalous terms in quantum gauge theory in the natural class of polynomials of differential forms. By using the appropriate cohomological and algebraic methods, we do it for all dimensions of spacetime and all structure groups with reductive Lie algebras.
\end{abstract}

\section{Introduction}

It is known that anomalous terms in quantum gauge theory (e.g. chiral anomalies, Schwinger terms) verify consistency equations [1,2] of cohomological nature [3]. The cohomology of interest is the local cohomology of the Becchi-Rouet-Stora (B.R.S) operator, [4].

An anomalous term $\Delta$ verifies a consistency equation: $\delta \Delta=0 . \Delta$, (the anomalous term), is the integral of a polynomial in the fields and their derivatives. However, solutions of the form $\Delta=\delta \Delta^{\prime}$, where $\Delta^{\prime}$ is a similar local expression, are considered as trivial; indeed, in the case of chiral anomalies or Schwinger terms, such trivial solutions may be cancelled by finite renormalization or by redefinition of the local currents respectively.

Setting $\Delta=\int Q$ leads, for $Q$, to the Eq. [5]

$$
\delta Q=d Q^{\prime}
$$

for some $Q^{\prime} ; d$ is the exterior differential on space-time. We shall say that $Q$ is a $\delta$-cocycle modulo $d$. If $\Delta$ is a trivial solution $\Delta=\delta \Delta^{\prime}$, then $Q$ reads

$$
Q=\delta L^{\prime}+d L
$$

for some $L^{\prime}$ and $L$; we say that such a $Q$ is a $\delta$-coboundary modulo $d$. As pointed out before, we are interested in solutions of $(*)$ modulo solutions of the form $(* *)$, i.e. in the $\delta$-cohomology modulo $d$.

More precisely, $d$ and $\delta$ act as antiderivations on polynomial functions of the gauge potential 1 -form $A$, the ghost field $\chi$ and their derivatives with values in differential forms on space-time $M . d$ is the exterior differential on space-time and 
$\delta$, (i.e. the B.R.S. operator), is defined by $\delta A=-d \chi-[A, \chi], \delta \chi=-1 / 2[\chi, \chi]$ and $d \delta+\delta d=0$, (which implies $\delta^{2}=0$ ).

Our purpose is to compute the $\delta$-cohomology modulo $d$ (i.e. possible anomalous terms). We shall do it in the algebra $\mathscr{B}(M, \mathfrak{g})$ of forms generated by exterior (pointwise in $M$ ) products of the components, in the Lie algebra $g$ of the structure group, of $A, \chi$ and their $d$ and $\delta$ differentials.

We shall proceed as follows:

In Sect. 2, we define a universal algebra $\mathscr{A}(\mathfrak{g})$ supporting the action of $d$ and $\delta$; this algebra only depends on the Lie algebra $\mathfrak{g}$.

In Sect. 3, we compute the $d$ and the $d+\delta$ cohomologies of $\mathscr{A}(\mathrm{g})$.

In Sect. 4, we compute the $\delta$-cohomology of $\mathscr{A}(\mathrm{g})$.

In Sect. 5, we consider the $\delta$-cohomology modulo $d$ of $\mathscr{A}(\mathfrak{g})$ and construct long exact sequences involving the $\delta$-cohomology and the $\delta$-cohomology modulo $d$ of $\mathscr{A}(\mathfrak{g})$.

In Sect. 6, we use the results of Sect. 5 to construct an exact couple relating the $\delta$-cohomology and the $\delta$-cohomology modulo $d$ of $\mathscr{A}(\mathfrak{g})$ and give a way to evaluate the $\delta$-cohomology modulo $d$ in terms of the associated spectral sequence.

In Sect. 7, we explicitly compute the $\delta$-cohomology modulo $d$ of $\mathscr{A}(\mathfrak{g})$ in the case where $g$ is reductive by using the general method of Sect. 6 .

In Sect. 8, we define the general notion of B.R.S. algebra; $\mathscr{A}(\mathrm{g})$ and $\mathscr{B}(M, \mathfrak{g})$ are such algebras. Moreover we describe the universal property of $\mathscr{A}(\mathrm{g})$ in this class. We finally show that the $\delta$-cohomology modulo $d$ of $\mathscr{B}(M, \mathrm{~g})$ is known from the one of $\mathscr{A}(\mathrm{g})$.

The content of Sects. 2-4 is summarized in our paper [6].

Finally, it is worth noticing here that some ideas of Weil are close to some developments given in this paper [7].

\section{The Universal B.R.S. Algebra of a Lie Algebra}

Let $g$ be a finite dimensional real Lie algebra and let $\left(E_{\alpha}\right)$ be a basis of $\mathfrak{g}$. Consider four copies $\mathfrak{g}_{A}^{*}, \mathfrak{g}_{F}^{*}, \mathfrak{g}_{x}^{*} \mathfrak{g}_{\varphi}^{*}$ of the dual space $\mathfrak{g}^{*}$ of $\mathfrak{g}$ with dual basis respectively denoted by $\left(A^{\alpha}\right),\left(F^{\alpha}\right),\left(\chi^{\alpha}\right)$ and $\left(\varphi^{\alpha}\right)$. Let $\mathscr{A}(\mathrm{g})$ be the free graded commutative algebra [8] generated by the $A^{\alpha}$ and $\chi^{\alpha}$ in degree one and the $F^{\alpha}$ and $\varphi^{\alpha}$ in degree two. In other words

$$
\mathscr{A}(\mathfrak{g})=\left(\Lambda \mathfrak{g}_{A}^{*}\right) \otimes\left(S \mathfrak{g}_{F}^{*}\right) \otimes\left(\Lambda \mathfrak{g}_{\chi}^{*}\right) \otimes\left(S \mathfrak{g}_{\varphi}^{*}\right),
$$

where $\Lambda \mathfrak{g}^{*}$ is the exterior algebra $\bigoplus_{n \in \mathbb{N}} \Lambda^{n} \mathfrak{g}^{*}$ of multilinear antisymmetric forms on $\mathfrak{g}$ graded by giving the degree $n$ to the elements of $\Lambda^{n} \mathrm{~g}^{*}$, where $S \mathrm{~g}^{*}$ is the algebra $\bigoplus_{n \in \mathbb{N}} S^{n} \mathrm{~g}^{*}$ of the polynomials on $\mathrm{g}$ evenly graded by giving the degree $2 n$ to the element of $S^{n} \mathrm{~g}^{*}$, and where $\otimes$ is the (Skew) tensor product of graded algebras [9]. On the space $\mathrm{g} \otimes \mathscr{A}(\mathrm{g})$, there is a natural bilinear bracket $[\cdot, \cdot]$ defined by $[X \otimes P$, $Y \otimes Q]=[X, Y] \otimes P \cdot Q$, for any $X, Y \in \mathfrak{g}$ and $P, Q \in \mathscr{A}(\mathfrak{g})$. Let us introduce the following elements of $\mathfrak{g} \otimes \mathscr{A}(\mathfrak{g}): A=\sum_{\alpha} E_{\alpha} \otimes A^{\alpha}, F=\sum_{\alpha} E_{\alpha} \otimes F^{\alpha}, \chi=\sum_{\alpha} E_{\alpha} \otimes \chi^{\alpha}$ and 
$\varphi=\sum_{\alpha} E_{\alpha} \otimes \varphi^{\alpha}$. Set, with the above notations $d A=F-1 / 2[A, A], d F=[F, A]$, $d \chi=\varphi, d \varphi=0$ and $\delta A=-\varphi-[A, \chi], \delta F=[F, \chi], \delta \chi=-1 / 2[\chi, \chi], \delta \varphi=[\varphi, \chi]$, and define $d A^{\alpha}, d F^{\alpha}, d \chi^{\alpha}, d \varphi^{\alpha}, \delta A^{\alpha}, \delta F^{\alpha}, \delta \chi^{\alpha}, \delta \varphi^{\alpha}$ in $\mathscr{A}(\mathfrak{g})$ by

$$
d A=\sum_{\alpha} E_{\alpha} \otimes d A^{\alpha}, \ldots, \delta \varphi=\sum_{\alpha} E_{\alpha} \otimes \delta \varphi^{\alpha}
$$

Then,

$$
\left(A^{\alpha}, F^{\alpha}, \chi^{\alpha}, \varphi^{\alpha}\right) \mapsto\left(d A^{\alpha}, d F^{\alpha}, d \chi^{\alpha}, d \varphi^{\alpha}\right)
$$

and

$$
\left(A^{\alpha}, F^{\alpha}, \chi^{\alpha}, \varphi^{\alpha}\right) \mapsto\left(\delta A^{\alpha}, \delta F^{\alpha}, \delta \chi^{\alpha}, \delta \varphi^{\alpha}\right)
$$

extend respectively uniquely as anti-derivations $d$ and $\delta$ of $\mathscr{A}(\mathfrak{g})$. One easily checks that $d$ and $\delta$ are of degree one and satisfy:

$$
d^{2}=0, \quad d \delta+\delta d=0 \text { and } \delta^{2}=0 .
$$

Thus $d, \delta$ and $d+\delta$ are three differentials on the graded algebra $\mathscr{A}(\mathfrak{g})$; we denote the corresponding graded algebras of cohomology by $H(d), H(\delta)$ and $H(d+\delta)$. We shall compute these algebras in the next sections. Let us now introduce an underlying bigraduation on $\mathscr{A}(\mathfrak{g})$ by giving to the $A^{\alpha}$ the bidegree $(1,0)$, to the $F^{\alpha}$ the bidegree $(2,0)$, to the $\chi^{\alpha}$ the bidegree $(0,1)$ and to the $\varphi^{\alpha}$ the bidegree $(1,1)$. So we have $\mathscr{A}(\mathfrak{g})=\bigoplus_{(r, s) \in \mathbb{N}^{2}} \mathscr{A}^{r, s}(\mathfrak{g})$ with $\mathscr{A}^{r, s}(\mathfrak{g}) \cdot \mathscr{A}^{k, \ell}(\mathfrak{g}) \subset \mathscr{A}^{r+k, s+\ell}(\mathfrak{g})$ and the (total) degree of an homogeneous element of bidegree $(r, s)$ is $r+s$. The differentials $d$ and $\delta$ are respectively of bidegrees $(1,0)$ and $(0,1)$, so the cohomology algebras $H(d)$ and $H(\delta)$ are bigraded algebras $H(d)=\bigoplus_{(r, s) \in \mathbb{N}^{2}} H^{r, s}(d)$ and $H(\delta)=\bigoplus_{(r, s) \in \mathbb{N}^{2}} H^{r, s}(\delta)$, whereas $H(d+\delta)$ is simply a graded algebra; $d=(d+\delta)_{(1,0)}, \delta=(d+\delta)_{(0,1)}$. We shall refer to the above structure as the universal B.R.S. algebra of $\mathfrak{g}$ and denote it by $\mathscr{A}(\mathfrak{g})$.

1.1. Remark. Notice that the subalgebra generated by the $A^{\alpha}$ and the $F^{\alpha}$ is stable by $d$ and that, equipped with $d$, it is just the Weil algebra of $g$. More generally for any real number $t \in \mathbb{R}$, one has the formula $[10,11]$

$$
(d+t \delta)(A+t \chi)+1 / 2[A+t \chi, A+t \chi]=F ;
$$

it follows that, for each $t \in \mathbb{R}$, the subalgebra $W_{t}(\mathfrak{g})$ of $\mathscr{A}(\mathrm{g})$ generated by the $A^{\alpha}$ $+t \chi^{\alpha}$ and the $F^{\alpha}$ equipped with the differential $d_{t}=d+t \delta$ is isomorphic, as graded differential algebra, to the Weil algebra $W(\mathfrak{g})$ of $\mathfrak{g}[12]$.

It will be convenient in the following, to extend the graduation of $\mathscr{A}(\mathfrak{g})$ to $\mathbb{Z}$ and the bigraduation to $\mathbb{Z} \times \mathbb{Z}$ by writing $\mathscr{A}^{k, \ell}(\mathfrak{g})=0$ whenever $k$ or $\ell$ is negative.

\section{The $d$ and the $d+\delta$ Cohomologies of $\mathscr{A}(\mathfrak{g})$}

We have the following theorem concerning $H(d)$ and $H(d+\delta)$.

3.1. Theorem. (a) The d-cohomology of $\mathscr{A}(\mathrm{g})$ is trivial, i.e. $H^{k, \ell}(d)=0$ for any positive integers $k, \ell$ such that $k+\ell \geqq 1$ and $H^{0,0}(d)=\mathbb{R}$. 
(b) The $(d+\delta)$-cohomology of $\mathscr{A}(\mathfrak{g})$ is trivial, i.e. $H^{n}(d+\delta)=0$ for any integer $n \geqq 1$ and $H^{0}(d+\delta)=\mathbb{R}$.

Proof. (a) It follows from $d A=F-1 / 2[A, A]$ and $d \chi=\varphi$ that $\mathscr{A}(\mathrm{g})$ is freely generated by the homogeneous elements $A^{\alpha}, \chi^{\alpha}, d A^{\alpha}, d \chi^{\alpha}$. Thus $(\mathscr{A}(\mathrm{g}), d)$ is the contractible algebra [8] generated by the $A^{\alpha}, \chi^{\alpha}$ in degree one and the $d A^{\alpha}, d \chi^{\alpha}$ in degree two which has trivial cohomology by standard arguments [8].

(b) It again follows from the definitions that $\mathscr{A}(\mathrm{g})$ is freely generated by the elements $A^{\alpha}, \chi^{\alpha},(d+\delta) A^{\alpha}$ and $(d+\delta) \chi^{\alpha}$, which are homogeneous for the total degree. Thus again $(\mathscr{A}(\mathfrak{g}), d+\delta)$ is contractible so we have: $H(d+\delta)=H^{0}(d+\delta)$ $=\mathbb{R}$.

3.2. Corollary. (a) Let $Q^{k, \ell} \in \mathscr{A}^{k, \ell}(\mathfrak{g})$ be such that there is a $Q^{k-1, \ell+1} \in \mathscr{A}^{k-1, \ell+1}(\mathfrak{g})$ such that $\delta Q^{k, \ell}+d Q^{k-1, \ell+1}=0$. Then, there is a $Q^{k-2, \ell+2} \in \mathscr{A}^{k-2, \ell+2}(\mathrm{~g})$ such that $\delta Q^{k-1, \ell+1}+d Q^{k-2, \ell+2}=0$.

(b) Let $Q^{k, \ell} \in \mathscr{A}^{k, \ell}(\mathfrak{g})$ be such that there are $L^{k, \ell-1} \in \mathscr{A}^{k, \ell-1}(\mathfrak{g})$ and $L^{k-1, \ell} \in \mathscr{A}^{k-1, \ell}(\mathfrak{g})$ with $Q^{k, \ell}=\delta L^{k, \ell-1}+d L^{k-1, \ell}$. Then $Q^{k, \ell}$ satisfies the assumption of (a) and any $Q^{k-1, \ell+1}$ as in (a) is of the form $Q^{k-1, \ell+1}=\delta L^{k-1, \ell}+d L^{k-2, \ell+1}$ for some $L^{k-2, \ell+1} \in \mathscr{A}^{k-2, \ell+1}(\mathrm{~g})$.

Proof. (a) Apply $\delta$ to $\delta Q^{k, \ell}+d Q^{k-1, \ell+1}=0$; it follows $\delta d Q^{k-1, \ell+1}$ $=d\left(-\delta Q^{k-1, \ell+1}\right)=0, \quad$ so $\quad \delta Q^{k-1, \ell+1}+d Q^{k-2, \ell+2}=0 \quad$ for some $Q^{k-2, \ell+2} \in \mathscr{A}^{k-2, \ell+2}(\mathrm{~g})$, in view of part (a) of the last theorem.

(b) We have $-\delta Q^{k, \ell}=+d \delta L^{k-1, \ell}$, so any $Q^{k-1, \ell+1}$ such that $\delta Q^{k, \ell}$ $+d Q^{k-1, \ell+1}=0$ satisfies $d\left(Q^{k-1, \ell+1}-\delta L^{k-1, \ell}\right)=0$. Thus again, by part (a) of the last theorem, $Q^{k-1, \ell+1}=\delta L^{k-1, \ell}+d L^{k-2, \ell+1}$ for some $L^{k-2, \ell+1} \in \mathscr{A}^{k-2, \ell+1}(\mathfrak{g})$.

3.3. Corollary. Let $P \in \mathscr{A}^{n+1,0}(\mathfrak{g})$ be such that $d P=0$ and $\delta P=0$. Then there are $Q^{n-p, p} \in \mathscr{A}^{n-p, p}(\mathfrak{g})$ for $0 \leqq p \leqq n$ such that we have: $P=d Q^{n, 0}, \delta Q^{n-p, p}$ $+d Q^{n-p-1, p+1}=0$ for $0 \leqq p \leqq n-1$ and $\delta Q^{0, n}=0$. Furthermore if $\widetilde{Q}^{n-p, p} \in \mathscr{A}^{n-p, p}(\mathfrak{g})$ with $0 \leqq p \leqq n$ satisfy the above relations, then there are $L^{n-p-1, p} \in \mathscr{A}^{n-p-1, p}(\mathfrak{g})$ for $0 \leqq p \leqq n-1$ such that $\widetilde{Q}^{n, 0}=Q^{n, 0}+d L^{n-1,0}, \widetilde{Q}^{n-p, p}$ $=Q^{n-p, p}+\delta L^{n-p, p-1}+d L^{n-p-1, p}$ for $1 \leqq p \leqq n-1$ and $\widetilde{Q}^{0, n}=Q^{0, n}+\delta L^{0, n-1}$.

Proof. We have by assumption $(d+\delta) P=0$; so part (b) of the last theorem implies that there is a $Q \in \mathscr{A}(\mathfrak{g})$ of total degree $n$ such that $P=(d+\delta) Q$. Thus writing $Q=\sum_{p=0}^{p=n} Q^{n-p, p}$ the $Q^{n-p, p}$ satisfy the statement. If $\tilde{Q}$ is another solution of $P=(d+\delta) \tilde{Q}$, then $(d+\delta)(\tilde{Q}-Q)=0 ;$ so again $\tilde{Q}=Q+(d+\delta) L$ for some $L=\sum_{p=0}^{n-1} L^{n-p-1, p}$, which proves the last part of the corollary.

\section{The $\delta$ Cohomology of $\mathscr{A}(\mathrm{g})$}

To compute $H(\delta)$ we first proceed to write the free-graded commutative differential algebra $(\mathscr{A}(\mathfrak{g}), \delta$ ) as a tensor product of a contractible algebra and a minimal algebra [8]. To do that we notice that $\mathscr{A}(\mathrm{g})$ is freely generated by the homogeneous elements $A^{\alpha}, \delta A^{\alpha}, \chi^{\alpha}, F^{\alpha}$; now the algebra generated by $A^{\alpha}, \delta A^{\alpha}$ is 
contractible whereas the algebra generated by the $\chi^{\alpha}, F^{\alpha}$ is isomorphic to $\left(S \mathfrak{g}_{\mathrm{F}}^{*}\right) \otimes\left(\Lambda \mathfrak{g}_{\chi}^{*}\right)$ as graded algebra and is minimal, (for $\delta$ ), in view of $\delta \chi=-1 / 2[\chi, \chi]$ and $\delta F=[F, \chi]$. The space $S \mathfrak{g}_{F}^{*}=S g^{*}$ is a graded $g$-module if we equip it with the representation of $\mathfrak{g}$ which comes, by symmetric tensor products, from the coadjoint representation of $\mathfrak{g}$ in $\mathfrak{g}^{*}$. One may clearly identify [13]

$$
S^{k} \mathrm{~g}^{*} \otimes \Lambda^{\ell} \mathrm{g}^{*} \simeq S^{k} \mathrm{~g}_{F}^{*} \otimes \Lambda^{\ell} \mathrm{g}_{\chi}^{*}
$$

with the space $C^{\ell}\left(\mathfrak{g}, S^{k} \mathfrak{g}^{*}\right)$ of $\ell$-cochains on $\mathfrak{g}$ with values in the $\mathfrak{g}$-module $S^{k} \mathfrak{g}^{*}$. It follows from the definitions that, under this identification, $\left(S \mathfrak{g}_{F}^{*} \otimes \Lambda \mathfrak{g}_{\chi}^{*}, \delta\right)$ identifies, as complex, with the complex $C^{*}\left(\mathfrak{g}, S_{\mathfrak{g}}^{*}\right)$ of cochains on $\mathfrak{g}$ with values in the graded $\mathfrak{g}$-module $\mathrm{Sg}^{*}$. Thus we have the following theorem.

4.1. Theorem. The $\delta$-cohomology of $\mathscr{A}(\mathfrak{g})$ coincides with the $\delta$-cohomology of the subalgebra generated by the $\chi^{\alpha}$ and the $F^{\alpha}$; as $\delta$-complex, this subalgebra is isomorphic to the complex $C^{*}\left(\mathfrak{g}, \mathrm{Sg}^{*}\right)$ of cochains on $\mathrm{g}$ with values in the $\mathrm{g}$-module $S_{\mathfrak{g}^{*}}$. So we have $H(\delta)=\bigoplus_{k, \ell} H^{2 k, \ell}(\delta)$ with $H^{2 k, \ell}(\delta)=H^{\ell}\left(\mathfrak{g}, S^{k} \mathfrak{g}^{*}\right)$ for all positive integers $k$ and $\ell$.

Let $\mathscr{I}_{S}(\mathfrak{g})\left(\subset S \mathfrak{g}^{*}\right)$ be the algebra of invariant polynomials on $\mathfrak{g}$. If $P \in \mathscr{I}_{S}^{k}(\mathfrak{g})$ and $Q \in \mathscr{I}_{S}^{\ell+1}(\mathfrak{g})$, then $P(F, \ldots, F) \in \mathscr{A}^{2 k, 0}(\mathfrak{g})$ and

$$
Q(\chi,[\chi, \chi], \ldots,[\chi, \chi]) \in \mathscr{A}^{0,2 \ell+1}(\mathfrak{g})
$$

are $\delta$-cocycles so the subalgebra $\mathscr{I}(F, \chi)$ generated by these $\delta$-cocycles when $P$ and $Q$ run over $\mathscr{I}_{S}(\mathfrak{g})$ consists of $\delta$-cocycles. Let us identify $\mathscr{I}(F, \chi)$ with the corresponding subspace of cocycles in $C^{*}\left(\mathfrak{g}, S \mathfrak{g}^{*}\right)$; then the linear span of the $Q(\chi,[\chi, \chi], \ldots,[\chi, \chi])$ as above identifies with the space of transgressive elements of $\Lambda \mathrm{g}^{*}$ [12]. In the case where $\mathrm{g}$ is a reductive Lie algebra, (i.e. $\mathrm{g}$ is the direct sum of a semi-simple Lie algebra and an abelian Lie algebra), it is known that the transgressive elements are the primitive elements and generate the subalgebra $\mathscr{I}_{\Lambda}(\mathfrak{g})$ of invariant forms in $\Lambda \mathfrak{g}^{*}$ (for adjoint action). It turns out that for reductive $\mathfrak{g}$, $\mathscr{I}_{\Lambda}(\mathfrak{g})$ is isomorphic to $H^{*}(\mathfrak{g})$ and that, on the other hand, we have for any semisimple graded $\mathfrak{g}$-module $\mathscr{M}, H^{*}(\mathfrak{g}, \mathscr{M})=\mathscr{M}^{I} \otimes H^{*}(\mathfrak{g})$, where $\mathscr{M}^{I}$ denotes the space of invariant elements of $\mathscr{M},[9,14]$. Therefore, by applying all that to $\mathrm{Sg}^{*}$ we obtain the following corollary.

4.2. Corollary. Let $\mathrm{g}$ be a reductive Lie algebra. Then the canonical projection from $\delta$-cocycles of $\mathscr{A}(\mathfrak{g})$ on $H(\delta)$ induces an isomorphism of the algebra $\mathscr{I}(F, \chi)$ on the algebra $H(\delta)$.

$\mathscr{I}(F, \chi) \simeq H(\delta)$ is an isomorphism of bigraded algebras.

4.3. Remarks. 1. The class of reductive Lie algebras is the appropriate class to write Yang-Mills lagrangians since it is only for a reductive Lie algebra $g$ that there exists a finite dimensional faithful representation of $g$ with a non-degenerate trace form, [9].

2. For any Lie algebra $\mathfrak{g}, \mathscr{I}(F, 0)$ is the set of all $\delta$-cocycles contained in $\bigoplus_{n \in \mathbb{N}} \mathscr{A}^{n, 0}(\mathfrak{g})$. Therefore any $\delta$-cocycle in $\mathscr{A}^{2 k+1,0}(\mathfrak{g})$ vanishes and any $\delta$-cocycle in 
$\mathscr{A}^{2 k, 0}(\mathfrak{g})$ is of the form $P(F, \ldots, F)$ for some invariant polynomial $P \in \mathscr{I}_{S}^{k}(\mathfrak{g})$. In particular, any $\delta$-cocycle in $\mathscr{A}^{n, 0}(\mathfrak{g})$ is $d$-closed.

4.4. Corollary. Let $Q^{2 k, 1} \in \mathscr{A}^{2 k, 1}(\mathfrak{g})$ be such that there is a $Q^{2 k-1,2} \in \mathscr{A}^{2 k-1,2}(\mathfrak{g})$ such that $\delta Q^{2 k, 1}+d Q^{2 k-1,2}=0$. Then, there is a unique $Q^{2 k+1,0} \in \mathscr{A}^{2 k+1,0}(\mathfrak{g})$ such that $\delta Q^{2 k+1,0}+d Q^{2 k, 1}=0$, and we have $d Q^{2 k+1,0}=P(F, \ldots, F)$ for a unique $P \in \mathscr{I}_{S}^{k+1}(\mathfrak{g})$.

Proof. We have $d \delta Q^{2 k, 1}=\delta\left(-d Q^{2 k, 1}\right)=0$, and, by the Theorem $4.1, H^{2 k+1,1}(\delta)$ $=H^{2 k+1,0}(\delta)=0$; so there is a unique $Q^{2 k+1,0}$ for which we have $\delta Q^{2 k+1,0}$ $+d Q^{2 k, 1}=0$. It follows that $\delta d Q^{2 k+1,0}=0$, and therefore $d Q^{2 k+1,0}=P(F, \ldots, F)$ for an invariant polynomial $P$ on $\mathfrak{g}$ of degree $k+1$.

4.5. Corollary. Let $\mathrm{g}$ be a semi-simple Lie algebra and let $n$ and $k$ be two integers such that $0 \leqq k \leqq 2 n+1$ and such that either $k \leqq 5$ or $n \leqq 3$. Then, if $Q^{2 n-k+1, k} \in \mathscr{A}^{2 n-k+1, k}(\mathfrak{g})$ is such that there is a $Q^{2 n-k, k+1} \in \mathscr{A}^{2 n-k, k+1}(\mathfrak{g})$ with $\delta Q^{2 n-k+1, k}+d Q^{2 n-k, k+1}=0$, there is a $P \in \mathscr{I}_{S}^{n+1}(\mathfrak{g})$ and there are $Q^{2 n-p+1, p} \in \mathscr{A}^{2 n-p+1, p}(\mathfrak{g})$ for $p<k$ such that $P(F, \ldots, F)=d Q^{2 n+1,0}$ and $\delta Q^{2 n-p+1, p}+d Q^{2 n-p, p+1}=0$ for $p \leqq k$.

Proof. A semi-simple Lie algebra has no non-trivial invariant linear form, i.e. $\mathscr{I}_{S}^{1}(\mathfrak{g})=0$, so it follows from the Corollary 4.2 , that, if $n$ and $k$ are as above, $H^{2 n+2-p, p}(\delta)=0$ for any $1 \leqq p \leqq k$. This implies the result by the same argument as before.

\section{The $\delta$-Cohomology Modulo $d$ of $\mathscr{A}(\mathfrak{g})$}

We now describe a more powerful way to look at the statements of Corollaries 3.2, $3.3,4.4$, and 4.5 and to go further.

Notice first that $d \delta+\delta d=0$ implies that $d \mathscr{A}(\mathfrak{g})$ is stable by $\delta$ and therefore that $(d \mathscr{A}(\mathfrak{g}), \delta)$ is a subcomplex of $(\mathscr{A}(\mathfrak{g}), \delta)$. The differential $\delta$ passes to the quotient and gives a differential, again denoted by $\delta$, on $\mathscr{A}(\mathfrak{g}) / d \mathscr{A}(\mathfrak{g})$. We call $\delta$-cochains modulo $d$ the elements of $\mathscr{A}(\mathfrak{g}) / d \mathscr{A}(\mathfrak{g})$; we write

$$
C^{k, \ell}(\delta, \bmod (d))=\mathscr{A}^{k, \ell}(\mathfrak{g}) / d \mathscr{A}^{k-1, \ell}(\mathfrak{g}),
$$

and define

$$
\bigoplus_{k, \ell} H^{k, \ell}(\delta, \bmod (d))=H(\delta, \bmod (d)),
$$

the $\delta$-cohomology modulo $d$ of $\mathscr{A}(\mathfrak{g})$, as the cohomology of $C(\delta, \bmod (d))$ $=(\mathscr{A}(\mathfrak{g}) / d \mathscr{A}(\mathfrak{g}), \delta)$. Let $p: \mathscr{A}(\mathfrak{g}) \rightarrow C(\delta, \bmod (d))$ be the canonical projection, we denote by $p^{\#}: H(\delta) \rightarrow H(\delta, \bmod (d))$ the corresponding linear mapping in cohomology. A cocycle of $C(\delta, \bmod (d))$ will be called a $\delta$-cocycle modulo $d$ and a coboundary of $C(\delta, \bmod (d))$ will be called a $\delta$-coboundary modulo $d$.

$Q^{k, \ell} \in \mathscr{A}^{k, \ell}(\mathrm{g})$ represents a $\delta$-cocycle modulo $d$, (i.e. $\delta p\left(Q^{k, \ell}\right)=0$ ), if and only if there is a $Q^{k-1, \ell+1} \in \mathscr{A}^{k-1, \ell+1}(\mathrm{~g})$ such that $\delta Q^{k, \ell}+d Q^{k-1, \ell+1}=0 ; Q^{k, \ell}$ represents a $\delta$-coboundary modulo $d$ if and only if there are $L^{k, \ell-1} \in \mathscr{A}^{k, \ell-1}(\mathfrak{g})$ and $L^{k-1, \ell} \in \mathscr{A}^{k-1, \ell}(\mathrm{g})$ such that $Q^{k, \ell}=\delta L^{k, \ell-1}+d L^{k-1, \ell}$. Now, Corollary 3.2(a) means that if $Q^{k, \ell}$ represents a $\delta$-cocycle modulo $d$ then $Q^{k-1, \ell+1}$ as above represents a 
$\delta$-cocycle modulo $d$ and Corollary 3.2 (b) implies that the class of $Q^{k-1, \ell+1}$ in $H^{k-1, \ell+1}(\delta, \bmod (d))$ is unique and does only depend on the class of $Q^{k, \ell}$ in $H^{k, \ell}(\delta, \bmod (d))$. We denote by

$$
\partial: H^{k, \ell}(\delta, \bmod (d)) \rightarrow H^{k-1, \ell+1}(\delta, \bmod (d))
$$

the corresponding linear mappings in $H(\delta, \bmod (d))$. In the same way, Corollary 3.3 means that we have linear mappings

$$
j^{n, p}: Z^{n+1,0}(d+\delta) \rightarrow H^{n-p, p}(\delta, \bmod (d))
$$

for $0 \leqq p \leqq n$ with $j^{n, p+1}=\partial \circ j^{n, p}$ for $1 \leqq p+1 \leqq n$, where $Z^{n+1,0}(d+\delta)$ is the space of $d$-closed $\delta$-cocycle in $\mathscr{A}^{n+1,0}(\mathfrak{g})$. In view of Remark $4.3-2, Z^{2 k+1,0}(d+\delta)=0$ and $Z^{2 k, 0}(d+\delta)$ is the set of $P(F, \ldots, F)$, where $P$ runs over the space of invariant polynomials of degree $k$ on $\mathfrak{g}$, so we identify $Z^{2 k, 0}(d+\delta)$ with $\mathscr{I}_{S}^{k}(\mathfrak{g})$ which we also identify with

$$
H^{2 k, 0}(\delta) \simeq H^{0}\left(\mathfrak{g}, S^{k} \mathfrak{g}^{*}\right) .
$$

Summarizing, we have the commutative diagram:

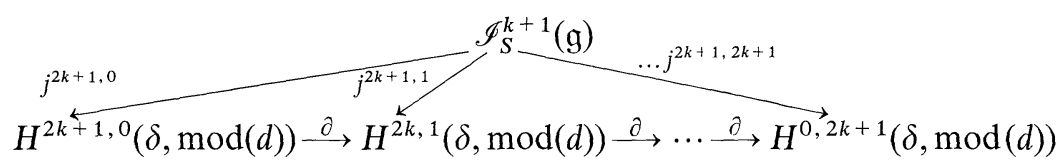

5.1. Remark. We have

$$
H^{0,2 k+1}(\delta, \bmod (d))=H^{0,2 k+1}(\delta)=H^{2 k+1}(\mathfrak{g})
$$

and one sees that

$$
j^{2 k+1,2 k+1}: \mathscr{I}_{S}^{k+1}(\mathfrak{g}) \rightarrow H^{2 k+1}(\mathfrak{g})
$$

is induced by the Cartan map $[9,12]$ of $\mathscr{I}_{S}^{k+1}(\mathrm{~g})$ on the transgressive elements of $\Lambda \mathfrak{g}^{*}$; it follows that $j^{2 k+1,2 k+1}$ vanishes on the elements of $\mathscr{I}_{S}^{k+1}(\mathrm{~g})$ which are decomposable [12]. In the case where $\mathfrak{g}$ is reductive the linear hull of the decomposable elements is exactly the kernel of $j^{2 k+1,2 k+1}$.

Corollary 4.4 tells us that the mappings

$$
\begin{gathered}
j^{2 k+1,1}: \mathscr{I}_{S}^{k+1}(\mathfrak{g}) \rightarrow H^{2 k, 1}(\delta, \bmod (d)), \\
j^{2 k+1,0}: \mathscr{I}_{S}^{k+1}(\mathfrak{g}) \rightarrow H^{2 k+1,0}(\delta, \bmod (d))
\end{gathered}
$$

and

$$
\partial: H^{2 k+1,0}(\delta, \bmod (d)) \rightarrow H^{2 k, 1}(\delta, \bmod (d))
$$

are all bijections. Finally, Corollary 4.5 means that, when $g$ is semi-simple, the maps

$$
j^{2 n+1, k}: \mathscr{I}_{S}^{n+1}(\mathfrak{g}) \rightarrow H^{2 n-k+1, k}(\delta, \bmod (d))
$$

and

$$
\partial: H^{2 n-k+2, k-1}(\delta, \bmod (d)) \rightarrow H^{2 n-k+1, k}(\delta, \bmod (d))
$$

are surjections whenever $0 \leqq k \leqq 2 n+1$ and either $k \leqq 5$ or $n \leqq 3$. 
5.2. Lemma. The mappings $Q^{k, \ell} \mapsto(-1)^{k} d Q^{k, \ell}$ from $\mathscr{A}^{k, \ell}(\mathrm{g})$ in $\mathscr{A}^{k+1, \ell}(\mathrm{g})$ induce, for $k+\ell \geqq 1$, isomorphisms of

$$
C^{k, \ell}(\delta, \bmod (d))=\mathscr{A}^{k, \ell}(\mathfrak{g}) / d \mathscr{A}^{k-1, \ell}(\mathfrak{g})
$$

on $d \mathscr{A}^{k, \ell}(\mathfrak{g})$ and permute with $\delta$; so we have

$$
H^{k, \ell}(\delta, \bmod (d))=H^{k+1, \ell}(d \mathscr{A}(\mathfrak{g}), \delta)
$$

for any integers $k, \ell$ with $k+\ell \leqq 1$.

Proof. This is an immediate consequence of Theorem 3.1 (a) and $\delta d=-d \delta$.

We have $d \mathscr{A}^{k, \ell}(\mathfrak{g}) \subset \mathscr{A}^{k+1, \ell}(\mathfrak{g})$, so, by the above lemma, we have injections

$$
i: C^{k, \ell}(\delta, \bmod (d)) \rightarrow \mathscr{A}^{k+1, \ell}(\mathfrak{g}),
$$

for $k+\ell \geqq 1$, which induce homomorphisms

$$
i^{\#}: H^{k, \ell}(\delta, \bmod (d)) \rightarrow H^{k+1, \ell}(\delta)
$$

in cohomology for $k+\ell \geqq 1$.

5.3. Proposition. We have $H^{0, \ell}(\delta, \bmod (d))=H^{0, \ell}(\delta)$, for any positive integer $\ell$ and we have, for $k \geqq 1$, long exact sequences, $\left(\mathscr{C}_{k}\right)$,

$\cdots \stackrel{i^{\#}}{\longrightarrow} H^{k, \ell}(\delta) \stackrel{p^{\#}}{\longrightarrow} H^{k, \ell}(\delta, \bmod (d)) \stackrel{\partial}{\longrightarrow} H^{k-1, \ell+1}(\delta, \bmod (d)) \stackrel{i^{\#}}{\longrightarrow} H^{k, \ell+1}(\delta) \stackrel{p^{\#}}{\longrightarrow} \cdots$

starting with

$$
0 \longrightarrow H^{1,0}(\delta) \stackrel{p^{*}}{\longrightarrow} H^{1,0}(\delta, \bmod (d)) \stackrel{\partial}{\longrightarrow} H^{0,1}(\delta, \bmod (d)) \stackrel{i^{*}}{\longrightarrow} \cdots,
$$

for $k=1$, and with

$$
0 \longrightarrow H^{k-1,0}(\delta, \bmod (d)) \stackrel{i^{\#}}{\longrightarrow} H^{k, 0}(\delta) \stackrel{p^{\#}}{\longrightarrow} H^{k, 0}(\delta, \bmod (d)) \stackrel{\partial}{\longrightarrow} \cdots
$$

for $k \geqq 2$.

Proof. $(d \mathscr{A}(\mathfrak{g}))^{0, \ell}=0$, sowe have $H^{0, \ell}(\delta, \bmod (d))=H^{0, \ell}(\delta)$. On the other hand, for any $k \geqq 1$, we have the short exact sequence of $\delta$-complexes,

$$
0 \longrightarrow(d \mathscr{A}(\mathfrak{g}))^{k, *} \stackrel{\subseteq}{\longrightarrow} \mathscr{A}^{k, *}(\mathfrak{g}) \stackrel{p}{\longrightarrow} C^{k, *}(\delta, \bmod (d)) \longrightarrow 0
$$

the corresponding long exact sequence of $\delta$-cohomologies just coincides with $\left(\mathscr{S}_{k}\right)$ by writing

$$
H^{k, l}(\delta, \bmod (d))=H^{k+1, \ell}(d \mathscr{A}(\mathfrak{g}), \delta)
$$

for $k+\ell \geqq 1$, and by using $(d \mathscr{A}(\mathfrak{g}))^{1,0}=d \mathscr{A}^{0,0}(\mathfrak{g})=0$. One verifies that $\partial$ as defined above is the connecting homomorphism of the long sequence $\left(\mathscr{S}_{k}\right)$.

By using $H^{2 k+1, \ell}(\delta)=0$ and $H^{2 k, \ell}(\delta)=H^{\ell}\left(\mathfrak{g}, S^{k} \mathfrak{g}^{*}\right)$, (see Theorem 4.1), one obtains the following theorem.

5.4. Theorem. (a) We have the following isomorphisms:

1) $H^{0, \ell}(\delta, \bmod (d)) \simeq H^{\ell}(\mathfrak{g}), \forall \ell \in \mathbb{N}$, (induced by $p^{\#}$ );

2) $H^{2 r+1,0}(\delta, \bmod (d)) \simeq H^{0}\left(\mathfrak{g}, S^{r+1} \mathfrak{g}^{*}\right)=\mathscr{I}_{S}^{r+1}(\mathfrak{g})$ and $H^{2 r+2,0}(\delta, \bmod (d))=0$, $\forall r \in \mathbb{N}$, (induced by $\left.i^{\#}\right)$; 

a).

3) $H^{2 r+1, \ell}(\delta, \bmod (d)) \simeq H^{2 r, \ell+1}(\delta, \bmod (d)), \forall r \in \mathbb{N}$ and $\forall \ell \in \mathbb{N}$, (induced by

(b) For any $r \in \mathbb{N}$, we have the long exact sequence $\left(\Im_{r}\right)$ :

$$
\begin{aligned}
& 0 \longrightarrow H^{2 r+1,1}(\delta, \bmod (d)) \stackrel{i^{\#}}{\longrightarrow} H^{1}\left(\mathfrak{g}, S^{r+1} \mathfrak{g}^{*}\right) \stackrel{p^{\#}}{\longrightarrow} H^{2 r+2,1}(\delta, \bmod (d)) \\
& \stackrel{\partial}{\longrightarrow} H^{2 r+1,2}(\delta, \bmod (d)) \stackrel{i^{\#}}{\longrightarrow} \cdots \stackrel{i^{\#}}{\longrightarrow} H^{\ell}\left(\mathfrak{g}, S^{r+1} \mathfrak{g}^{*}\right) \\
& \stackrel{p^{\#}}{\longrightarrow} H^{2 r+2, \ell}(\delta, \bmod (d)) \stackrel{\partial}{\longrightarrow} H^{2 r+1, \ell+1}(\delta, \bmod (d)) \\
& \stackrel{i^{\#}}{\longrightarrow} H^{\ell+1}\left(\mathfrak{g}, S^{r+1} \mathfrak{g}^{*}\right) \stackrel{p^{\#}}{\longrightarrow} \cdots .
\end{aligned}
$$

Notice that (apart from the isomorphism 1 which is trivial) the isomorphisms 2 and 3 are easy to prove directly.

5.5. Corollary. Let $\mathrm{g}$ be a semi-simple Lie algebra. Then, we have the isomorphisms:

$$
\begin{aligned}
\mathscr{I}_{S}^{r+2}(\mathfrak{g}) & \simeq H^{2 r+3,0}(\delta, \bmod (d)) \simeq H^{2 r+2,1}(\delta, \bmod (d)) \\
& \simeq H^{2 r+1,2}(\delta, \bmod (d)) \simeq H^{2 r, 3}(\delta, \bmod (d)),
\end{aligned}
$$

for $\quad r \in \mathbb{N}$. Furthermore, $H^{2 r+2,0}(\delta, \bmod (d)), \quad H^{2 r+1,1}(\delta, \bmod (d)) \quad$ and $H^{2 r, 2}(\delta, \bmod (d))$ vanish.

Proof. This follows immediately from Theorem 5.4 by using $H^{1}\left(\mathfrak{g}, S^{k} \mathfrak{g}^{*}\right)=0$ and $H^{2}\left(\mathrm{~g}, S^{k} \mathrm{~g}^{*}\right)=0$ (see in the proof of Corollary 4.5).

5.6. Corollary. $H^{k, \ell}(\delta, \bmod (d))=0$ for $\ell>\operatorname{dim}(\mathfrak{g})$.

Proof. We have $\Lambda^{\ell} \mathfrak{g}^{*}=0$ for $\ell>\operatorname{dim}(\mathfrak{g})$. It follows that we also have $H^{\ell}\left(\mathfrak{g}, S^{r} \mathfrak{g}^{*}\right)=0$ for $\ell>\operatorname{dim}(g)$, so that by Theorem 5.4 (part a.3 and part b) $\partial$ induces isomorphisms

$$
H^{k, \ell}(\delta, \bmod (d)) \simeq H^{k-1, \ell+1}(\delta, \bmod (d))
$$

for any $\ell>\operatorname{dim}(\mathfrak{g})$. This implies $H^{k, \ell}(\delta, \bmod (d))=0$ for $\ell>\operatorname{dim}(\mathfrak{g})$, since $\partial^{k+1} H^{k, \ell}(\delta, \bmod (d))=0$.

\section{The Exact Couple Relating $H(\delta, \bmod (d))$ and $H^{*}\left(\mathfrak{g}, S \mathfrak{g}^{*}\right)$}

6.1. Let us recall that an exact couple of vector spaces, [15], is an exact triangle of linear mappings $a, b, c$

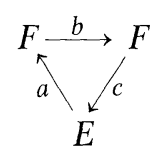

involving two vector spaces $E$ and $F$. Given such an exact couple, one can construct another one,

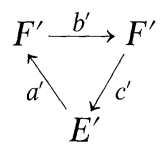

called the derived exact couple by the following procedure: One takes $F^{\prime}=b(F)$ and $b^{\prime}=b\left\lceil b(F): F^{\prime} \rightarrow F^{\prime}\right.$. From exactness at $E$, it follows that $d=c \circ a$ is a differential on 
$E$, (i.e. $d^{2}=0$ ), so the homology $H(E, d)$ is well defined; one takes $E^{\prime}=H(E, d)$. From exactness at $F$, it follows that $a$ maps the $d$-closed elements of $E$ into $b(F)$ $=F^{\prime}$ and the $d$-exact elements of $E$ on $0 \in F^{\prime} ; a^{\prime}$ is the induced map of $E^{\prime}$ in $F^{\prime}$. Again by exactness, $c$ maps $F$ into $d$-closed elements of $E$ and the class $[c(f)] \in E^{\prime}$ does only depend on $b(f) \in F^{\prime}$, for $f \in F$; $c^{\prime}$ is the corresponding map of $F^{\prime}$ in $E^{\prime}$. One verifies that the triangle of linear mappings $a^{\prime}, b^{\prime}, c^{\prime}$ so defined is again exact.

By induction, one defines, for any integer $r$, the $r^{\text {th }}$ derived exact couple

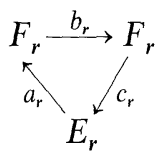

writing $E_{0}=E, F_{0}=F, a_{0}=a, b_{0}=b, c_{0}=c$ and $E_{r+1}=E_{r}^{\prime}, F_{r+1}=F_{r}^{\prime}, a_{r+1}=a_{r}^{\prime}$, $b_{r+1}=b_{r}^{\prime}, c_{r+1}=c_{r}^{\prime}$. Setting $d_{r}=c_{r} \circ a_{r}$, we have $E_{r+1}=H\left(E_{r}, d_{r}\right)$, so $\left(E_{r}, d_{r}\right)_{r \in \mathbb{N}}$ is a spectral sequence associated to the exact couple. We have, in view of $\operatorname{kerb}_{r}=a_{r}\left(E_{r}\right)$ and $\operatorname{Imb}_{r}=F_{r+1}$, the following isomorphisms:

$$
F_{r}=b^{r}(F) \simeq a_{r}\left(E_{r}\right) \oplus F_{r+1}=a_{r}\left(E_{r}\right) \oplus b^{r+1}(F), \quad r \in \mathbb{N} .
$$

It follows that we also have:

$$
F \simeq\left(\bigoplus_{r=0}^{r=n} a_{r}\left(E_{r}\right)\right) \oplus b^{n+1}(F), \quad \forall n \in \mathbb{N} .
$$

Let us now come back to our original problem. It is clear from (a). 3 in Theorem 5.4 that one may replace in the long sequences $\left(\Theta_{r}\right)$ of 5.4 the $H^{2 r+1, \ell}(\delta, \bmod (d))$ by the $H^{2 r, \ell+1}(\delta, \bmod (d))$. Thus we surely have an exact couple involving $H^{\mathrm{ev}, *}(\delta, \bmod (d))$ and $H^{*}\left(\mathfrak{g}, S \mathfrak{g}^{*}\right)$, (ev. stands for even here); one must however carefully check what is going on for the lowest degrees in $(r, \ell)$ by using the results 5.4.(a)-1 and 5.4.(a)-2. It is easy to see that, in order that it works, one has to replace $H^{0,0}(\delta, \bmod (d))$ and $H^{0}(\mathfrak{g})$ by 0 ; so we define $\mathbb{Z}^{2}$-graded spaces $H_{+}^{\text {ev., } *}(\delta, \bmod (d))$ and $H_{+}^{*}\left(\mathfrak{g}, S \mathfrak{g}^{*}\right)$ by

$$
\left(H_{+}^{\mathrm{ev},}, *(\delta, \bmod (d))\right)^{r, s}=H^{2 r, s}(\delta, \bmod (d))
$$

and

$$
\left(H_{+}^{*}\left(\mathfrak{g}, S \mathfrak{g}^{*}\right)\right)^{r, s}=H^{s}\left(\mathfrak{g}, S^{r} \mathfrak{g}^{*}\right)
$$

for $r \geqq 0, s \geqq 0$ and $r+s \geqq 1$ and

$$
\left(H_{+}^{\text {ev. }, *}(\delta, \bmod (d))\right)^{r, s}=\left(H_{+}^{*}\left(\mathfrak{g}, S \mathfrak{g}^{*}\right)\right)^{r, s}=0
$$

otherwise. Let

$$
p_{0}: H_{+}^{*}\left(\mathfrak{g}, S \mathfrak{g}^{*}\right) \rightarrow H_{+}^{\mathrm{ev} .}, *(\delta, \bmod (d))
$$

be defined by

$$
p_{0}=p^{\#}: H^{s}\left(\mathfrak{g}, S^{r} \mathfrak{g}^{*}\right) \rightarrow H^{2 r, s}(\delta, \bmod (d))
$$

for $r \geqq 0, s \geqq 0, r+s \geqq 1$ and by $p_{0}: 0 \rightarrow 0$ otherwise; let

$$
i_{0}: H_{+}^{\text {ev. }, *}(\delta, \bmod (d)) \rightarrow H_{+}^{*}\left(\mathfrak{g}, S \mathfrak{g}^{*}\right)
$$


be the canonical extension to $(r, s) \in \mathbb{Z}^{2}$ of

$$
i_{0}=i^{\#} \circ \partial^{-1}: H^{2 r, s+1}(\delta, \bmod (d)) \rightarrow H^{s}\left(\mathfrak{g}, S^{r+1} \mathfrak{g}^{*}\right)
$$

for $r \geqq 0$ and $s \geqq 0$. Finally $\partial^{2}$ extends canonically as

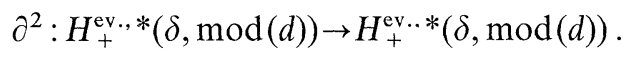

With these notations, we may formulate a part of 5.4 as follows.

6.2. Theorem. We have the following exact triangle $\left(\mathscr{E}_{0}\right)$

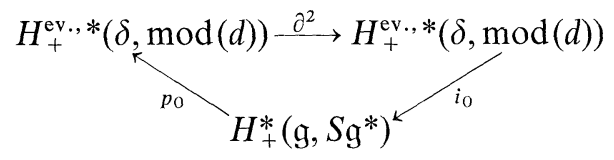

Thus $\left(\mathscr{E}_{0}\right)$ is an exact couple of vector spaces relating the $\delta$ cohomology modulo $d$ of $\mathscr{A}(\mathfrak{g})$ to the cohomology of $\mathfrak{g}$ with values in the polynomials on $\mathfrak{g}$. Let $\left(E_{r}, d_{r}\right)_{r \in \mathbb{N}}$ be the associated spectral sequence starting with $E_{0}=H_{+}^{*}\left(\mathrm{~g}, S \mathrm{~g}^{*}\right)$ and $d_{0}=i_{0} \circ p_{0}$; the $r^{\text {th }}$ derived exact couple $\left(\mathscr{E}_{r}\right)$ may be written as

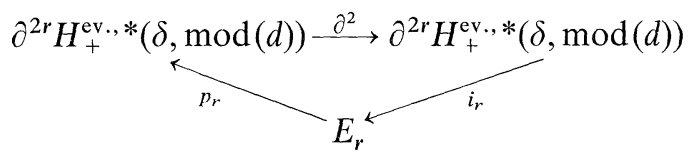

It follows, as above, that we have the isomorphisms

$$
H_{+}^{\text {ev., } *}(\delta, \bmod (d)) \simeq\left(\bigoplus_{r=0}^{r=n} p_{r}\left(E_{r}\right)\right) \oplus \partial^{2(n+1)} H_{+}^{\text {ev. }, *}(\delta, \bmod (d)),
$$

$(\forall n \in \mathbb{N})$, and since $\partial^{2 n}(x)=0$ for $n$ greater than some finite $n_{0}(x)$ for each $x$, we have the isomorphism:

$$
H_{+}^{\mathrm{ev} .,} *(\delta, \bmod (d)) \simeq \bigoplus_{r \in \mathbb{N}} p_{r}\left(E_{r}\right)
$$

Therefore, in order to compute the $\delta$ cohomology $\bmod (d)$, it is sufficient to compute the $E_{r}$, the kernels of $p_{r}$ and, eventually, to give a procedure to construct $\delta$-cocycles modulo $d$ corresponding to elements of $\bigoplus_{r \in \mathbb{N}} E_{r}$; we shall do that in the case where $g$ is a reductive Lie algebra in the next section.

\section{The Case of a Reductive Lie Algebra $\mathfrak{g}$}

In this section, $\mathfrak{g}$ denotes a reductive Lie algebra. Let us recall that, in this case, $H^{*}(\mathfrak{g})$ identifies with the algebra $\mathscr{I}_{A}(\mathfrak{g})$ of invariant forms on $g$ and that this algebra is freely generated, as graded commutative algebra, by any basis of homogeneous primitive invariant forms, [9, 16]; furthermore, any homogeneous primitive invariant form is of odd degree so $\mathscr{I}_{\Lambda}(\mathfrak{g})$ coincides with the exterior algebra over the space $P$ of primitive invariant forms on $\mathfrak{g}$, i.e. $\mathscr{I}_{\Lambda}(\mathfrak{g})=\Lambda P$. Let $P^{2 k+1}$ be the space of primitive invariant forms of degree $2 k+1 ; P=\underset{k \geqq 0}{\bigoplus} P^{2 k+1}$, and since 
$\operatorname{dim}(P)$ is the rank of $\mathfrak{g}$, the spaces $P^{2 k+1}$ all vanish when $k$ is greater than some finite integer $r_{M}(\mathfrak{g})$. As pointed out in Remark 5.1, the Cartan map $\varrho: \mathscr{I}_{S}^{k+1}(\mathfrak{g})$ $\rightarrow \mathscr{I}_{\Lambda}^{2 k+1}(\mathfrak{g})$ is induced by $j^{2 k+1,2 k+1}$ via the identifications

$$
H^{0,2 k+1}(\delta, \bmod (d))=H^{2 k+1}(\mathfrak{g})=\mathscr{I}_{\Lambda}^{2 k+1}(\mathfrak{g}) ;
$$

its image coincides with $P^{2 k+1}$, as is well known, $[9,12]$, i.e. we have $\varrho\left(\mathscr{I}_{S}(\mathrm{~g})\right)=P$ and $\varrho\left(\mathscr{J}_{S}^{k+1}(\mathfrak{g})\right)=P^{2 k+1}$. We choose once and for all a transgression $\tau$, i.e. a linear mapping $\tau: P \rightarrow \mathscr{I}_{S}(\mathrm{~g})$, such that $\tau\left(P^{2 k+1}\right) \subset \mathscr{I}_{S}^{k+1}(\mathrm{~g})$ and such that $\varrho \circ \tau$ is the identity mapping of $P$ on itself. Then $\tau$ induces isomorphisms $P^{2 k+1} \simeq \tau\left(P^{2 k+1}\right)$ and, as is well known $[9,17]$, the image by $\tau$ of a homogeneous basis of $P$ is a free system of generators of the commutative algebra $\mathscr{I}_{S}(\mathfrak{g})$ which therefore coincides with the symmetric algebra over $\tau(P)$; i.e. we have $\mathscr{I}_{S}(\mathfrak{g})=S \tau(P) \simeq S P$. Remembering that $H^{*}\left(\mathfrak{g}, S \mathfrak{g}^{*}\right)$ identifies with $\mathscr{I}_{S}(\mathfrak{g}) \otimes \mathscr{I}_{A}(\mathfrak{g})$, we write:

$$
H^{*}\left(\mathfrak{g}, S_{\mathfrak{g}}^{*}\right)=(S \tau(P)) \otimes(\Lambda P) \simeq(S P) \otimes(\Lambda P) .
$$

Let us introduce the subspaces $P_{r}=\bigoplus_{k \geq_{r}} P^{2 k+1}$ of $P\left(=P_{0}\right)$, and define the subalgebras $\mathscr{I}_{r}$ and $E_{r}=\mathscr{I}_{r}^{+}$of

$$
\mathscr{I}_{S}(\mathfrak{g}) \otimes \mathscr{I}_{\Lambda}(\mathfrak{g}), \quad(r \in \mathbb{N}),
$$

by

$$
\mathscr{I}_{r}=\left(S \tau\left(P_{r}\right)\right) \otimes\left(\Lambda P_{r}\right)
$$

and

$$
E_{r}=\underset{m+n \geqq 1}{\bigoplus}\left(S^{m} \tau\left(P_{r}\right)\right) \otimes\left(\Lambda^{n} P_{r}\right)\left(=\mathscr{I}_{r}^{+}\right) .
$$

We have:

$$
E_{r}=E_{r+1} \oplus\left(\bigoplus_{m+n \geqq 1}\left(S^{m} \tau\left(P^{2 r+1}\right)\right) \otimes\left(\Lambda^{n} P^{2 r+1}\right)\right) \otimes \mathscr{I}_{r+1}=E_{r+1} \oplus E_{r}^{r}
$$

(i.e. $E_{r}^{r}$ involves at least one primitive element of degree $2 r+1$ ). The identification

$$
H^{*}\left(\mathfrak{g}, S \mathfrak{g}^{*}\right)=\mathscr{I}_{S}(\mathfrak{g}) \otimes \mathscr{I}_{\Lambda}(\mathfrak{g})=\mathscr{I}_{0}
$$

leads to $H_{+}^{*}\left(\mathfrak{g}, S \mathrm{~g}^{*}\right)=E_{0}$; furthermore we have $E_{r}=0$ for $r>r_{M}(\mathrm{~g})$. Let $d_{r}$ be the unique antiderivation of $\mathscr{I}_{r}$ such that

$$
d_{r}\left(\mathbb{1} \otimes P_{r+1}\right)=0, \quad d_{r}\left(\tau\left(P_{r}\right) \otimes \mathbb{1}\right)=0
$$

and

$$
d_{r}(\mathbb{1} \otimes \alpha)=\tau(\alpha) \otimes \mathbb{1} \quad \text { for } \quad \alpha \in P^{2 r+1} .
$$

Then we have $d_{r}^{2}=0, d_{r}\left(E_{r}\right) \subset E_{r}$, so the homology $H\left(E_{r}, d_{r}\right)$ is well defined.

7.1. Lemma. We have $E_{r+1}=H\left(E_{r}, d_{r}\right)$ for $r \in \mathbb{N}$, i.e. the sequence $\left(E_{r}, d_{r}\right)_{r \in \mathbb{N}}$ is a spectral sequence.

Proof. We have $d_{r}\left(E_{r+1}\right)=0$ and $d_{r}\left(E_{r}^{r}\right) \subset E_{r}^{r}$, so all we have to prove is that the homology $H\left(E_{r}^{r}, d_{r}\right)$ vanishes. Define $d_{r}^{\prime}$ to be the unique antiderivation of $\mathscr{I}_{r}$ such 
that

$$
d_{r}^{\prime}\left(\mathbb{1} \otimes P_{r}\right)=0, \quad d_{r}^{\prime}\left(\tau\left(P_{r+1}\right) \otimes \mathbb{1}\right)=0
$$

and

$$
d_{r}^{\prime}(\tau(\alpha) \otimes \mathbb{1})=\mathbb{1} \otimes \alpha \quad \text { for } \quad \alpha \in P^{2 r+1} .
$$

Then $d_{r}^{\prime}\left(E_{r}^{r}\right) \subset E_{r}^{r}$, and the derivation $d_{r} d_{r}^{\prime}+d_{r}^{\prime} d_{r}$ coincides on

$$
\left(S^{m} \tau\left(P^{2 r+1}\right)\right) \otimes\left(\Lambda^{n} P^{2 r+1}\right) \otimes \mathscr{I}_{r+1}
$$

with the multiplication by the number $n+m$ so, since

$$
E_{r}^{r}=\left(\bigoplus_{n+m \geqq 1}\left(S^{m} \tau\left(P^{2 r+1}\right)\right) \otimes\left(\Lambda^{n} P^{2 r+1}\right)\right) \otimes \mathscr{I}_{r+1},
$$

any $d_{r}$-closed element of $E_{r}^{r}$ is $d_{r}$-exact, which achieves the proof.

We shall show that $\left(E_{r}, d_{r}\right)_{r \in \mathbb{N}}$ is the spectral sequence associated to the exact couple $\left(\mathscr{E}_{0}\right)$ of Sect. 6 , and this is why we use this notation; for that, we need the following lemma.

7.2. Lemma. (Generalised "transgression" lemma). Let $X \in E_{r}$, then, there are $Q_{k} \in \mathscr{A}(\mathfrak{g})$ for $k=1,2, \ldots, 2 r+2$ such that we have: $d X+\delta Q_{1}=0, d Q_{k}+\delta Q_{k+1}=0$, for $1 \leqq k \leqq 2 r$, and $d Q_{2 r+1}+\delta Q_{2 r+2}=d_{r} X$. In other words, there is an element $\alpha$ of $H_{+}^{\text {ev. }} *(\delta, \bmod (d))$ such that $d_{r} X=i_{0}(\alpha)\left(=i^{\#} \circ \partial^{-1}(\alpha)\right)$ and $\partial^{2 r} \alpha=p_{0}(X)\left(=p^{\#}(X)\right)$; (Take $\alpha$ to be the class of $Q_{2 r}$ as above in $H_{+}^{\mathrm{ev} .}, *(\delta, \bmod (d))$ ).

Proof. It is clearly sufficient to consider monomials

$$
X=\prod_{i=1}^{i=m} \tau\left(\zeta_{i}\right)(F) \omega_{0}(\chi) \ldots \omega_{n}(\chi)
$$

where the $\zeta$ and the $\omega$ are homogeneous primitive forms of degrees greater than or equal to $2 r+1$. Introduce $\mathfrak{g}$-invariant $L_{p}$ satisfying [12]

$$
\tau\left(\omega_{p}\right)(F)=d L_{p}(A ; F)=(d+\delta) L_{p}(A+\chi ; F) ;
$$

we have: $L_{p}(\chi, 0)=\omega_{p}(\chi)$,

$$
\begin{aligned}
(d+\delta) \prod_{i=1}^{i=m} \tau\left(\zeta_{i}\right)(F) L_{0}(A+\chi ; F) \ldots L_{n}(A+\chi ; F) \\
\quad=\sum_{p=0}^{p=n}(-1)^{p} \prod_{i=1}^{i=m} \tau\left(\zeta_{i}\right)(F) \tau\left(\omega_{p}\right)(F) L_{0}(A+\chi ; F) \ldots L_{n}(A+\chi ; F)
\end{aligned}
$$

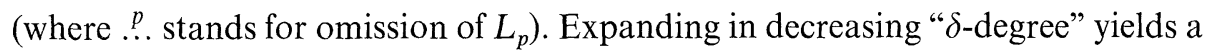
number of equations. The first $2 r+3$ equations give $\delta X=0$ and the equations of the lemma with explicit $Q_{k}$; as expected, the last equation (the $2 r+3^{\text {th }}$ ) gives $d_{r} X$ as defined before, i.e.

$$
\begin{aligned}
d Q_{2 r+1}+\delta Q_{2 r+2} & =\sum_{\left\{p \text { such that } \omega_{p} \in P^{2 r+1}\right\}}(-1)^{p} \prod_{i=1}^{i=m} \tau\left(\zeta_{i}\right)(F) \tau\left(\omega_{p}\right)(F) \omega_{0} \stackrel{p}{p} . \omega_{n} \\
& =d_{r} X . \quad \square
\end{aligned}
$$


7.3. Remarks. 1. In this proof, one explicitly sees that the image of the Cartan map $\varrho$ is the space $P$ of primitive elements of $\mathscr{I}_{\Lambda}(\mathfrak{g})$. By similar arguments, one easily sees that the decomposable elements of $\mathscr{I}_{S}(\mathfrak{g})$ are in the kernel of $\varrho$.

2. Notice that if $\zeta \in P^{2 s+1}$ with $s<r$, we may apply the same trick to $\tau(\zeta)(F) X$ as we did to $X$ in the proof of the lemma, but the point is that $p_{0}(\tau(\zeta)(F) X)=0$, i.e. $\tau(\zeta)(F) X=d A+\delta B$ for some elements $A$ and $B$ of $\mathscr{A}(\mathfrak{g})$.

We are now ready to identify $\left(E_{r}, d_{r}\right)_{r \in \mathbb{N}}$ with the spectral sequence associated with $\left(\mathscr{E}_{0}\right)$.

7.4. Theorem. We have the following exact triangles $\left(\mathscr{E}_{r}\right)$

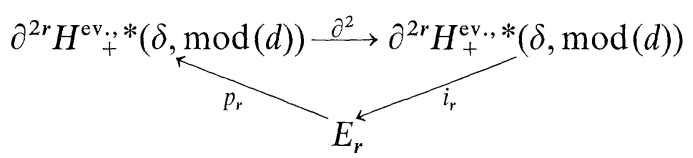

where $p_{r}$ is induced, by restriction to $E_{r}$, by $p_{0}$ and where $i_{r}\left(\partial^{2 r} \alpha\right)$ is the component of $i_{0}(\alpha)$ on $E_{r}$ in the direct sum decomposition $E_{0}=H_{+}^{*}\left(\mathfrak{g}, S \mathfrak{g}^{*}\right)=E_{r} \oplus \bigoplus_{s=0}^{s=r-1} E_{s}^{s}$. Furthermore we have $d_{r}=i_{r} \circ p_{r}$, so $\left(\mathscr{E}_{r}\right)$ identifies with the $r^{\text {th }}$ derived exact couple of $\left(\mathscr{E}_{0}\right)$ and $\left(E_{r}, d_{r}\right)_{r \in \mathbb{N}}$ is the associated spectral sequence.

Proof. Let $X$ be in $E_{r}$. Choose $\alpha$ as in 7.2; so

$$
i_{r} \circ p_{r}(X)=i_{r}\left(\partial^{2 r} \alpha\right)=\operatorname{proj}_{E_{r}} i_{0}(\alpha)=\operatorname{proj}_{E_{r}} d_{r} X=d_{r} X \text {. }
$$

Thus we have $d_{r}=i_{r} \circ p_{r}$. Then, by $7.1, E_{r+1}=H\left(E_{r}, d_{r}\right)$ so, by induction on $r,\left(\mathscr{E}_{r}\right)$ identifies with the $r^{\text {th }}$ derived exact couple of $\left(\mathscr{E}_{0}\right)$.

Together with the results of Sect. 6, this theorem implies the following result.

7.5. Corollary. We have the isomorphism:

$$
H_{+}^{\mathrm{ev} .}, *(\delta, \bmod (d)) \simeq \bigoplus_{r=0}^{r=r_{M}(\mathrm{~g})} p_{r}\left(E_{r}\right)=\bigoplus_{r=0}^{r=r_{M}(\mathrm{~g})} p^{\#}\left(E_{r}\right)
$$

Moreover, we have: $\partial^{2 r} H_{+}^{\mathrm{ev} .} * *(\delta, \bmod (d))=0$ for $r>r_{M}(\mathfrak{g})$.

Proof. The first part is clear. For the last part we remark that if $r>r_{M}(\mathfrak{g}), \quad E_{r}=0$ so

$$
\partial^{2}: \partial^{2 r} H_{+}^{\mathrm{ev} .}, *(\delta, \bmod (d)) \rightarrow \partial^{2 r} H_{+}^{\mathrm{ev} .}, *(\delta, \bmod (d))
$$

is an isomorphism, and therefore

$$
\partial^{2 r} H_{+}^{\mathrm{ev} .,} *(\delta, \bmod (d))=\partial^{2(r+k)} H_{+}^{\mathrm{ev} .}, *(\delta, \bmod (d))
$$

for any $k \geqq 0$; the result then follows from the fact that for any $x \in H_{+}^{\text {ev., } *}(\delta, \bmod (d))$, there is an integer $n$ such that $\partial^{2 n} x=0$.

7.6. Proposition. We have: $\operatorname{ker}\left(p_{r}\right)=\bigoplus_{k=r}^{k=r_{M}(\mathrm{~g})} \operatorname{Im}\left(d_{k}\right)$.

Proof. Let $X \in E_{r}$ satisfying $p_{r}(X)=0$; then $d_{r} X=0$. Since $E_{r+1}=H\left(E_{r}, d_{r}\right)$, we have $X=d_{r} Y+Z$ with $Z \in E_{r+1}$. Thus we have $p_{r+1}(Z)=p_{r}(Z)=p_{r}(X)-p_{r} \circ d_{r}(Y)=0$, so $X \in \operatorname{Im}\left(d_{r}\right) \oplus \operatorname{ker}\left(p_{r+1}\right)$. The result follows by induction. 
In the above result, (and in the proof), we explicitly use $\operatorname{Im}\left(d_{r+k}\right) \subset E_{r+k}^{r+k}$ and $E_{r}$ $=\bigoplus_{k \geq 0} E_{r+k}^{r+k}$

As a consequence of the last proposition and Lemma 7.1, we have the following isomorphisms:

$$
\begin{aligned}
p_{r}\left(E_{r}\right) \simeq E_{r} / \operatorname{ker}\left(p_{r}\right) & =\bigoplus_{s=r}^{s=r_{M}(\mathfrak{g})}\left(\mathscr{N}_{s} / d_{s}\left(\mathscr{N}_{s}\right)\right) \otimes \mathscr{I}_{s+1} \\
& \simeq \bigoplus_{s=r}^{s=r_{M}(\mathfrak{g})} d_{s}\left(\mathcal{N}_{s}\right) \otimes \mathscr{I}_{s+1}
\end{aligned}
$$

with

$$
\mathscr{N}_{s}=\bigoplus_{m+n \geqq 1} S^{m} \tau\left(P^{2 s+1}\right) \otimes \Lambda^{n} P^{2 s+1}
$$

7.7. Remark. The isomorphism in 7.5 may now be realized by the following procedure. Given a homogeneous basis $\left(\omega_{p}\right)$ of $P$, choose $\mathfrak{g}$-invariant $L_{p}(A ; F)$ such that

$$
\tau\left(\omega_{p}\right)(F)=d L_{p}(A ; F)=(d+\delta) L_{p}(A+\chi ; F) ;
$$

then, using the construction given in the proof of 7.2 one obtains a linear mapping

$$
\psi: \bigoplus_{r=0}^{r=r_{M}(\mathrm{~g})} E_{r} \rightarrow H_{+}^{\mathrm{ev} .,} *(\delta, \bmod (d))
$$

Choose, for each $r$, a supplementary $\mathscr{K}_{r}$ in $E_{r}$ to $\operatorname{ker}\left(p_{r}\right) ; \psi$ restricted to $\bigoplus_{r=0}^{r=r_{M}(\mathfrak{g})} \mathscr{K}_{r}$ gives, when combined with

$$
\bigoplus_{r \geqq 0} \mathscr{K}_{r} \simeq \underset{r \geqq 0}{\bigoplus_{r}} p_{r}\left(E_{r}\right)
$$

a realization of the isomorphism of 7.5 .

\section{B.R.S. Algebras}

8.1. Definition. Let $\mathfrak{g}$ be a Lie algebra. We define a B.R.S. algebra over $g$ to be a pair $(\mathscr{B}, \omega)$, where $\mathscr{B}$ is a bigraded differential algebra, i.e. $\mathscr{B}=\bigoplus_{(r, s) \in \mathbb{N}^{2}} \mathscr{B}^{r, s}$ is equipped with two anti-derivations $d$ and $\delta$ satisfying

$$
d \mathscr{B}^{r, s} \subset \mathscr{B}^{r+1, s}, \quad \delta \mathscr{B}^{r, s} \subset \mathscr{B}^{r, s+1}, \quad d^{2}=0,
$$

$\delta^{2}=0$ and $d \delta+\delta d=0$, and where $\omega$ is an element of $\mathfrak{g} \otimes\left(\mathscr{B}^{1,0} \oplus \mathscr{B}^{0,1}\right)$ satisfying

$$
(d+\delta) \omega+1 / 2[\omega, \omega] \in \mathscr{B}^{2,0} \quad(\mathscr{R}) .
$$

$\omega \in \mathfrak{g} \otimes\left(\mathscr{B}^{1,0} \oplus \mathscr{B}^{0,1}\right)$ will be called the algebraic connection, or simply the connection, of the B.R.S. algebra. When there is no ambiguity on the connection, we shall speak of the B.R.S. algebra $\mathscr{B}$. In the relation $(\mathscr{R}),[\cdot, \cdot]$ is the natural bracket on $\mathfrak{g} \otimes \mathscr{B}$ and $d+\delta$ is defined on $\mathfrak{g} \otimes \mathscr{B}$ by $(d+\delta) X \otimes P=X \otimes(d+\delta) P$ for any $X \in \mathfrak{g}$ and $P \in \mathscr{B}$. 
Given two B.R.S. algebras $(\mathscr{B}, \omega)$ and $\left(\mathscr{B}^{\prime}, \omega^{\prime}\right)$ over $\mathfrak{g}$, a homomorphism of B.R.S. algebras of $(\mathscr{B}, \omega)$ in $\left(\mathscr{B}^{\prime}, \omega^{\prime}\right)$ is a homomorphism $f: \mathscr{B} \rightarrow \mathscr{B}$ ' of bigraded differential algebras satisfying $\bar{f}(\omega)=\omega^{\prime}$, where $\bar{f}: \mathfrak{g} \otimes \mathscr{B} \rightarrow \mathfrak{g} \otimes \mathscr{B}^{\prime}$ is defined by

$$
\bar{f}(X \otimes P)=X \otimes f(P), \quad \forall X \in \mathfrak{g} \quad \text { and } \quad \forall P \in \mathscr{B} .
$$

If we equip $\mathscr{A}(\mathfrak{g})$ with the connection $A+\chi$, it is clear that it is a B.R.S. algebra over $g$. The name of universal B.R.S. algebra of $g$ comes from the following obvious lemma.

8.2. Lemma. For any B.R.S. algebra $\mathscr{B}$ over $\mathfrak{g}$, there is a unique homomorphism $f_{\mathscr{B}}: \mathscr{A}(\mathfrak{g}) \rightarrow \mathscr{B}$ of B.R.S. algebras.

In other words, there is a unique homomorphism of bigraded differential algebras, $f_{\mathscr{B}}: \mathscr{A}(\mathfrak{g}) \rightarrow \mathscr{B}$, mapping $A+\chi$ on the connection $\omega$ of $\mathscr{B}\left(\bar{f}_{\mathscr{B}}(A+\chi)=\omega\right)$. We call $f_{\mathscr{B}}$ the canonical homomorphism of $\mathscr{A}(\mathfrak{g})$ in $\mathscr{B}$.

8.3. Example. The algebra $\mathscr{B}(M, \mathfrak{g})$ of Sect. 1 . Let $G$ be a Lie group with Lie algebra $\mathfrak{g}$ and consider the gauge theory over space-time $M$ with structure group $G$. Let $d$ be the exterior differential of forms on space-time and $\delta$ be the usual B.R.S. operator. We consider the components in $g$ of generic gauge potential as 1 -forms on $M$ which anticommute with the components in $g$ of the ghost field and denote by $\mathscr{B}(M, \mathfrak{g})$ the algebra generated, (by pointwise exterior product on space-time), by these 1 -forms, the components on $\mathfrak{g}$ of the ghost field and their $d$ and $\delta$ differentials. This is a bigraded algebra $\mathscr{B}(M, \mathfrak{g})=\underset{r, s \leqq 0}{\bigoplus_{\mathfrak{B}}} \mathscr{B}^{r, s}(M, \mathfrak{g})$, where $r$ is the degree in form on $M,(d$-degree $)$, and where $s$ is the degree in the ghost field ( $\delta$-degree). Thus $\mathscr{B}(M, \mathfrak{g})$ is a bigraded differential algebra and, if we define $\omega$ to be the sum of the gauge potential and the ghost field, then $(\mathscr{R})$ is satisfied so $\mathscr{R}(M, \mathfrak{g})$ is a B.R.S. algebra over $\mathfrak{g}$. The canonical homomorphism of $\mathscr{A}(\mathfrak{g})$ in $\mathscr{B}(M ; \mathfrak{g})$ is described by the following procedure: The value at $x \in M$ for gauge potential $A(x)$ and ghost field $\chi(x)$ of the element of $\mathscr{B}(M, \mathfrak{g})$ corresponding to an element $P(A, F, \chi, \varphi)$ of $\mathscr{A}(\mathfrak{g})$ is obtained by replacing in $P$ the generators $A^{\alpha}, F^{\alpha}, \chi^{\alpha}, \varphi^{\alpha}$ of $\mathscr{A}(\mathfrak{g})$ by the (components in $\mathfrak{g}) A^{\alpha}(x), F^{\alpha}(x)=(d A(x)+1 / 2[A(x), A(x)])^{\alpha}, \chi^{\alpha}(x)$, $d \chi^{\alpha}(x)$.

8.4. Remark. As far as we are concerned with anomalous terms and consistency equations, the ghost field may be identified with the identity mapping of $\mathfrak{g}$ on itself considered as an element of $\underline{\mathfrak{g}} \otimes \Lambda^{1} \underline{\mathfrak{g}}^{*} \mathrm{C} \mathfrak{g} \otimes \Lambda \underline{\mathfrak{g}}^{*}$, where $\mathfrak{g}$ denotes the Lie algebra of the gauge group (i.e. here the smooth functions of $\bar{M}$ in $\mathrm{g}$ ).

8.5. Proposition. The canonical homomorphism of $\mathscr{A}(\mathfrak{g})$ in $\mathscr{B}(M, \mathfrak{g})$ is surjective and induces, by restriction, an isomorphism of vector spaces of $\mathscr{A}^{r, s}(\mathfrak{g})$ on $\mathscr{B}^{r, s}(M, \mathfrak{g})$ for $r \leqq \operatorname{dim}(M)$.

Proof. It is clear, from the definition of $\mathscr{B}(M, \mathfrak{g})$, that the canonical homomorphism is surjective from $\mathscr{A}(\mathfrak{g})$ on $\mathscr{B}(M, \mathfrak{g})$. A basis of $\mathscr{A}^{r, s}(\mathfrak{g})$ consists of the

$$
\begin{gathered}
A^{\alpha_{1}} \ldots A^{\alpha_{a}}\left(F^{\beta_{1}}\right)^{m_{1}} \ldots\left(F^{\beta_{b}}\right)^{m_{b}} \chi^{\gamma_{1}} \ldots \chi^{\gamma_{c}}\left(d \chi^{\delta_{1}}\right)^{n_{1}} \ldots\left(d \chi^{\delta_{d}}\right)^{n_{d}}, \\
a+2 \sum_{i=1}^{i=b} m_{i}+\sum_{j=1}^{j=d} n_{j}=r, \quad c+\sum_{j=1}^{j=d} n_{j}=s
\end{gathered}
$$


and where

$$
\alpha_{1}<\alpha_{2}<\ldots<\alpha_{a}, \quad \beta_{1}<\beta_{2}<\ldots<\beta_{b}, \quad \gamma_{1}<\gamma_{2}<\ldots<\gamma_{c}
$$

and $\delta_{1}<\ldots<\delta_{d}$ with $\alpha_{a}, \beta_{b}, \gamma_{c}$ and $\delta_{d}$ smaller than $\operatorname{dim}(\mathfrak{g})$. Thus all we have to show is that, if $r \leqq \operatorname{dim}(M)$, the functionals of the gauge potential and the ghost field corresponding to the above basis are linearly independent. To show that, it is sufficient to construct, for $\alpha_{\mu}, \beta_{v}, \gamma_{\lambda}, \delta_{\sigma}, m_{i}, n_{j}$ as above with $r \leqq \operatorname{dim}(M)$, a gauge potential $A(x)$ and a ghost field $\chi(x)$ such that at point $x_{0} \in M$ we have

$$
\begin{aligned}
A^{\alpha_{1}}\left(x_{0}\right) \ldots A^{\alpha_{a}}\left(x_{0}\right)\left(F^{\beta_{1}}\left(x_{0}\right)\right)^{m_{1}} \ldots\left(F^{\beta_{b}}\left(x_{0}\right)\right)^{m_{b}} \\
\\
\cdot \chi^{\gamma_{1}}\left(x_{0}\right) \ldots \chi^{\gamma_{c}}\left(x_{0}\right)\left(d \chi^{\delta_{1}}\left(x_{0}\right)\right)^{n_{1}} \ldots\left(d \chi^{\delta_{d}}\left(x_{0}\right)\right)^{n_{d}} \neq 0,
\end{aligned}
$$

and all the other products vanish. To construct such a configuration, we notice that given a $g$-valued 1 -form $A_{0}$ at $x_{0}$ and a $g$-valued two form $F_{0}$ at $x_{0}$ there is a $\mathfrak{g}$-valued 1-form $A(x)$ on $M$ such that $A\left(x_{0}\right)=A_{0}$ and $d A\left(x_{0}\right)+1 / 2\left[A\left(x_{0}\right), A\left(x_{0}\right)\right]$ $=F_{0}$, and that similar consideration applies to $\chi(x), d \chi(x)$. Thus, there is a $A(x)$ and a $\chi(x)$, such that $A^{\alpha_{1}}\left(x_{0}\right)=d x^{1}, \ldots, A^{\alpha_{a}}\left(x_{0}\right)=d x^{a}$ the other components of $A\left(x_{0}\right)$ vanish,

$$
F^{\beta_{1}}\left(x_{0}\right)=\frac{1}{m_{1} !} \sum_{k=a+1}^{k=a+m_{1}} d x^{k} \Lambda d x^{k+m_{1}}, \ldots, F^{\beta_{b}}\left(x_{0}\right)=\frac{1}{m_{b} !} \sum_{k=a+\sum_{b=1} \sum_{i=1} 2 m_{l}+1}^{k=a+\sum_{\substack{1 \\ \sum}}^{b-1} 2 m_{\imath}+m_{b}} d x^{k} \Lambda d x^{k+m_{b}},
$$

the other components of $F\left(x_{0}\right)$ vanish, $\chi^{\gamma_{1}}\left(x_{0}\right)=\chi^{\gamma_{1}}, \ldots, \chi^{\gamma_{c}}\left(x_{0}\right)=\chi^{\gamma_{c}}$ the other components of $\chi\left(x_{0}\right)$ vanish,

$$
d \chi^{\delta_{1}}\left(x_{0}\right)=\frac{1}{n_{1} !} \sum_{k=a+2 \Sigma m_{i}+1}^{a+2 \Sigma m_{i}+n_{1}} d x^{k} \chi_{k}^{\delta_{1}}, \ldots, d \chi^{\delta_{d}}\left(x_{0}\right)=\frac{1}{n_{d} !} \sum_{a+2 \Sigma m_{i}+{ }^{d} \Sigma^{1} n_{j}+1}^{a+2 \Sigma m_{i}+\Sigma n_{j}} d x^{k} \chi_{k}^{\delta_{d}}
$$

the other components of $d \chi\left(x_{0}\right)$ vanish, where the $x^{k}$ are coordinates around $x_{0}$ in $M$ and where the $\chi^{\gamma_{\lambda}}, \chi_{k}^{\delta_{\sigma}}$ are linearly independent. Such a configuration satisfies the above conditions.

8.6. Corollary. The canonical homomorphism of $\mathscr{A}(\mathfrak{g})$ in $\mathscr{B}(M, \mathfrak{g})$ induces isomorphisms of their $\delta$-cohomology and of their $\delta$-cohomology modulo $d$ in bidegrees $(r, s)$ for $r \leqq \operatorname{dim}(M)$.

Thus the computation of the $\delta$-cohomology modulo $d$ of $\mathscr{A}(\mathfrak{g})$ done in the preceding sections applies directly to the computation of anomalous terms in $\mathscr{B}(M, \mathfrak{g})$.

\section{Conclusion}

We have computed all possible anomalous terms which are (exterior) products of gauge potential 1-forms, ghost field and their $d$ and $\delta$ differentials. It would be desirable to extend these results to more general expressions containing arbitrary derivatives of the fields since, in principle, such expressions could occur in some 
models (although no non-trivial examples are known up to now). We shall apply our results to specific examples in a forthcoming publication.

Acknowledgements. We would like to thank R. Stora for his suggestions and his constant encouragement. We also thank L. Baulieu, D. Dhar, B. Julia, J. Madore, and J. B. Zuber for discussions.

\section{References}

1. Wess, J., Zumino, B.: Consequences of anomalous Ward identities. Phys. Lett. 37 B, 95 (1971)

2. Faddeev, L.D.: Operator anomaly for the Gauss law. Phys. Lett. 145 B, 81 (1984)

3. Becchi, C., Rouet, A., Stora, R.: Renormalization of gauge theories. Ann. Phys. (N.Y.) 98, 287 (1976)

4. Bonora, L., Cotta-Ramusino, P.: Some remarks on B.R.S. transformations, anomalies and the cohomology of the Lie algebra of the group of gauge transformations. Commun. Math. Phys. 87, 589 (1983)

5. Dixon, J.A.: Cohomology and renormalization of gauge theories, I, II, III. (unpublished) quoted in: Stora, R.: Continuum gauge theories. In: New developments in quantum field theory and statistical mechanics. Lévy, M., Mitter, P. (eds.) New York: Plenum 1977

6. Dubois-Violette, M., Talon, M., Viallet, C.M.: Results on B.R.S. cohomologies in gauge theory. Phys. Lett. 158B, 231 (1985)

7. Weil, A.: Géométrie différentielle des espaces fibrés, [1949e], In: Oeuvres scientifiques I (1926-1951). Berlin, Heidelberg, New York: Springer 1979

8. Sullivan, D.: Infinitesimal computations in topology. Publ. I.H.E.S. 47, 269 (1977)

9. Greub, W., Halperin, S., Vanstone, R.: Connections, curvature and cohomology. Vol. III. New York: Academic Press 1976

10. Stora, R.: Algebraic structure and topological origin of anomalies. In: Recent progress in gauge theories, Lehmann, G., et al. (eds.), New York: Plenum 1984

Zumino, B.: Chiral anomalies and differential geometry. In: Relativity, groups and topology II. DeWitt, B.S., Stora, R. (eds.). Amsterdam: North-Holland 1984

11. Baulieu, L.: Anomalies and gauge symmetry. Nucl. Phys. B 241, 557 (1984);

Baulieu, L., Thierry-Mieg, J.: Algebraic structure of quantum gravity and the classification of the gravitational anomalies. Phys. Lett. 145 B, 53 (1984)

12. Cartan, H.: Notion d'algèbre différentielle; application aux groupes de Lie et aux variétés où opère un groupe de Lie, and La transgression dans un groupe de Lie et dans un espace fibré principal. In: Colloque de topologie, (Bruxelles 1950). Paris: Masson 1951

13. Chevalley, C., Eilenberg, S.: Cohomology of Lie groups and Lie algebras. Trans. Am. Math. Soc. 63, 85 (1948)

14. Hochschild, G., Serre, J.P.: Cohomology of Lie algebras. Ann. Math. 57, 591 (1953)

15. MacLane, S.: Homology. Grundlehren der mathematischen Wissenschaften. Berlin, Heidelberg, New York: Springer 1963

16. Koszul, J.L.: Homologie et cohomologie des algèbres de Lie. Bull. Soc. Math. Fr. 78, 65 (1950)

17. Chevalley, C.: Invariants of finite groups generated by reflections. Am. J. Math. 77, 778 (1955)

Communicated by A. Jaffe

Received June 11, 1985; in revised form June 28, 1985 